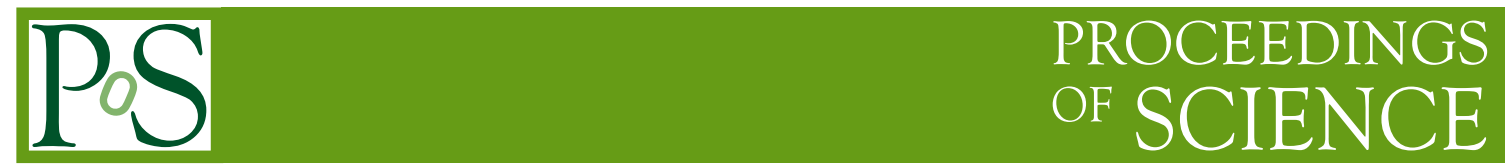

\title{
Electromagnetic currents from chiral EFT
}

\section{S. Kölling ${ }^{* a b}$}

${ }^{a}$ Forschungszentrum Jülich, Institut für Kernphysik (IKP-3) and Jülich Center for Hadron Physics, D-52425 Jülich, Germany

${ }^{b}$ Helmholtz-Institut für Strahlen- und Kernphysik (Theorie) and Bethe Center for Theoretical Physics,

Universität Bonn, D-53115 Bonn, Germany

E-mail: s.koellingefz-juelich.de

We derive the leading two-pion exchange contributions to the two-nucleon electromagnetic current operator in the framework of chiral effective field theory using the method of unitary transformation. Explicit results for the current in coordinate space are given.

6th International Workshop on Chiral Dynamics, CD09

July 6-10, 2009

Bern, Switzerland

${ }^{*}$ Speaker. 


\section{Introduction}

Chiral effective field theory (EFT) provides a systematic and model-independent framework to analyze low-energy hadronic processes. In particular, nuclear forces have been successfully derived [1]]. However, knowledge of the consistent electromagnetic exchange current operator is needed to study, for example, electron and photon few-nucleon reactions with momentum transfer of order $M_{\pi}$.

To the best of our knowledge, no applications have so far been performed to electron and photon inelastic few-nucleon reactions with the momentum transfer of the order $M_{\pi}$ where a lot of experimental data are available, see [2] for a recent review article on the theoretical achievements in this field based on conventional framework. Recent progress in the accurate description of the two[3, 4] and more-nucleon systems [5] within the chiral EFT, see also [6] and references therein, gives a strong motivation to apply this framework to the abovementioned processes. This requires the knowledge of the consistent electromagnetic exchange current operator for non-vanishing values of the photon momentum. While the leading two-nucleon contributions to the exchange current arise from one-pion exchange and are well known, the corrections at the one-loop level have not yet been completely worked out.

Pastore et al. [7, 8] have recently performed an important step towards the description of the exchange current operator. They calculate the electromagnetic current operator at leading one-loop order based on time-ordered perturbation theory.

In this contribution, we discuss the two-pion exchange current operator at leading one-loop order in the framework of unitary transformation. Since we use a completely different formalism, our results provide a non-trivial check of the results of Pastore et al. Further, we also present results for the exchange current density which, to the best of our knowledge, have not been presented before. The results are given in extremely compact formulae in configuration space. An expression in momentum space and a more details can be found in [9].

This manuscript is structured as follows. In section 2 we briefly review the method of unitary transformation and discuss how nuclear currents can be calculated. Results in configuration space are given in section 3 . We end with a summary and outlook.

\section{Nuclear currents using the method of unitary transformation}

We begin with a brief reminder about the method of unitary transformation, applied in the calculation of nuclear potentials, for details see [1].

Following Okubo [10] the unitary transformation $U$ can be parameterized as

$$
U=\left(\begin{array}{cc}
\eta\left(1+A^{\dagger} A\right)^{-1 / 2} & -A^{\dagger}\left(1+A A^{\dagger}\right)^{-1 / 2} \\
A\left(1+A^{\dagger} A\right)^{-1 / 2} & \lambda\left(1+A A^{\dagger}\right)^{-1 / 2}
\end{array}\right),
$$

in terms of the operator $A$ with the property $A=\lambda A \eta$, where we introduced projection operators $\eta(\lambda)$ on the purely nucleonic (the remaining) part of the Fock space satisfying $\eta^{2}=\eta, \lambda^{2}=\lambda$, $\eta \lambda=\lambda \eta=0$ and $\lambda+\eta=\mathbf{1}$. The operator $A$ has to be chosen in a way that the transformed Hamiltonian $\tilde{H} \equiv U^{\dagger} H U$ is block-diagonal in the $\eta$ - and $\lambda$ - subspaces. 
In Ref. [11], a convenient formulation of the power counting has been presented. The lowmomentum dimension $v$ of the effective potential, $V, V \sim \mathscr{O}(Q / \Lambda)^{v}$ with $Q$ and $\Lambda$ refering to the soft and hard scales of the order of the pion and $\rho$-meson masses, respectively, is given (modulo the normalization constant -2 ) by the overall inverse mass dimension of the coupling constants entering the expression for $V$ :

$$
v=-2+\sum V_{i} \kappa_{i}, \quad \kappa_{i}=d_{i}+\frac{3}{2} n_{i}+p_{i}-4
$$

Here, where $V_{i}$ is the number of vertices of type $i$ while $d_{i}, n_{i}$ and $p_{i}$ refer to the number of derivatives or $M_{\pi}$-insertions, nucleon field and pion field operators, respectively. Further, $\kappa_{i}$ is simply the canonical field dimension of a vertex of type $i$ (up to the additional constant -4 ). Writing the effective chiral Hamiltonian $H$ as

$$
H=\sum_{\kappa=1}^{\infty} H^{(\kappa)},
$$

the operator $A$ can be calculated recursively from the requirement that the transformed Hamiltonian is block-diagonal,

$$
\begin{aligned}
A & =\sum_{\alpha=1}^{\infty} A^{(\alpha)} \\
A^{(\alpha)} & =\frac{1}{E_{\eta}-E_{\lambda}} \lambda\left[H^{(\alpha)}+\sum_{i=1}^{\alpha-1} H^{(i)} A^{(\alpha-i)}-\sum_{i=1}^{\alpha-1} A^{(\alpha-i)} H^{(i)}-\sum_{i=1}^{\alpha-2} \sum_{j=1}^{\alpha-j-1} A^{(i)} H^{(j)} A^{(\alpha-i-j)}\right] \eta
\end{aligned}
$$

Here, $E_{\eta}\left(E_{\lambda}\right)$ refers to the free energy of nucleons (nucleons and pions) in the state $\eta(\lambda)$. It is important to emphasize that Eq. (2.1) does not provide the most general parametrization of the unitary operator. Moreover, as found in Ref. [11], the subleading contributions to the threenucleon force obtained using the parametrization in Eq. (2.1) cannot be renormalized. To restore renormalizability at the level of the Hamilton operator additional unitary transformation $U^{\prime}$ in the $\eta$-subspace of the Fock space had to be employed, $\eta U^{\prime} \eta U^{\prime \dagger} \eta=\eta U^{\prime \dagger} \eta U^{\prime} \eta=\eta$, whose explicit form at lowest non-trivial order is given in that work.

It is, in principle, straightforward to extend this formalism to low-energy electromagnetic reactions such as e.g. electron scattering off light nuclei. Here and in what follows, we restrict ourselves to the one-photon-exchange approximation to the scattering amplitude. The effective nuclear current operator $\eta J_{\mathrm{eff}}^{\mu}(x) \eta$ acting in the $\eta$-space is then defined according to

$$
\left\langle\Psi_{f}\left|J^{\mu}(x)\right| \Psi_{i}\right\rangle=\left\langle\phi_{f}\left|\eta U^{\prime \dagger} \eta U^{\dagger} J^{\mu}(x) U \eta U^{\prime} \eta\right| \phi_{i}\right\rangle \equiv\left\langle\phi_{f}\left|\eta J_{\mathrm{eff}}^{\mu}(x) \eta\right| \phi_{i}\right\rangle,
$$

where $\eta\left|\phi_{i, f}\right\rangle=\eta U^{\prime \dagger} \eta U^{\dagger}\left|\Psi_{i, f}\right\rangle$ denote the transformed states and we have omitted the components $\lambda\left|\phi_{i, f}\right\rangle$ which is justified as long as one stays below the pion production threshold. In the above expression, $J^{\mu}(x)$ denotes the hadronic current density which enters the effective Lagrangian $\mathscr{L}_{\pi N \gamma}$ describing the interaction of pions and nucleons with an external electromagnetic field $\mathscr{A}^{\mu}$ and is given by

$$
J^{\mu}(x)=\partial_{v} \frac{\partial \mathscr{L}_{\pi N \gamma}}{\partial\left(\partial_{\nu} \mathscr{A}_{\mu}\right)}-\frac{\partial \mathscr{L}_{\pi N \gamma}}{\partial \mathscr{A}_{\mu}} .
$$

Notice that contrary to the Hamilton operator, the unitarily transformed current does, in general, not have the block-diagonal form, i.e. $\eta U^{\dagger} J^{\mu}(x) U \lambda \neq 0$. Again, it is important to realize that 
the above definition of $\eta J_{\text {eff }}^{\mu}(x) \eta$ does not fully incorporate the freedom in the choice of unitary transformations. Thus, one might expect that this formulation yields the effective current operator which is not renormalizable by a redefinition of the low-energy constants (LECs) entering the underlying Lagrangian. Indeed, renormalizability of the effective current operator implies highly non-trivial constraints in the case of one-pion exchange contributions at the one-loop level since all $\beta$-functions of the LECs $l_{i}$ from $\mathscr{L}_{\pi}\left[12,13\right.$, and $d_{i}$ from $\mathscr{L}_{\pi N}[14,15]$ are fixed. We have verified that the ultraviolet divergences entering the expressions for the one-pion exchange contributions using the formulation based on the $\mathscr{A}^{\mu}$-independent unitary transformation as described above can indeed not be completely removed by the redefinition of the corresponding LECs. Thus, a more general parametrization of the unitary transformation is required in order to restore renormalizability of the nuclear current. This can be achieved if one allows for the unitary operator to depend explicitly on the external electromagnetic field, $U\left(\mathscr{A}^{\mu}\right)$. The operator $U\left(\mathscr{A}^{\mu}\right)$ then has to be chosen in such a way that the transformed Hamiltonian $U^{\dagger}\left(\mathscr{A}^{\mu}\right) H_{\pi N \gamma} U\left(\mathscr{A}^{\mu}\right)$ is block-diagonal (with respect to the $\eta$ - and $\lambda$-spaces) and coincides with the one given in Ref. 111] when the external electromagnetic field is switched off. The effective nuclear current operator $\eta J_{\text {eff }}^{\mu}(x) \eta$ in this more general formulation receives additional contributions which are not included in Eq. (2.5) and result from $\mathscr{A}^{\mu}$-dependent pieces of $U\left(\mathscr{A}^{\mu}\right)$ in the expression $U^{\dagger}\left(\mathscr{A}^{\mu}\right) H_{\pi N \gamma} U\left(\mathscr{A}^{\mu}\right)$ whose form is determined by renormalizability of the resulting nuclear current operator. These additional terms in $\eta J_{\text {eff }}^{\mu}(x) \eta$ are found to have no effect on the two-pion exchange current and will be discussed in detail in a separate publication [16] devoted to the one-pion exchange contributions. Finally, we emphasize that the power counting employed in the present work implies the following restrictions on the photon momentum $k$ in the two-nucleon rest frame

$$
|\vec{k}| \sim \mathscr{O}\left(M_{\pi}\right), \quad k^{0} \sim \mathscr{O}\left(\frac{M_{\pi}^{2}}{m}\right) \ll M_{\pi},
$$

where $M_{\pi}$ and $m$ refer to the pion and nucleon masses, respectively. For the kinematics with $k^{0} \sim \mathscr{O}\left(M_{\pi}\right)$, one will have to systematically keep track of the new soft momentum scale $\sqrt{M_{\pi} m}$. This goes beyond the scope of the present work.

For the calculation of the leading two-pion exchange two-nuclear current operator in the present work we only need the leading pion and pion-nucleon terms in the effective Lagrangian

$$
\begin{aligned}
& \mathscr{L}_{\pi \pi}^{(2)}=\frac{F_{\pi}^{2}}{4} \operatorname{tr}\left[D_{\mu} U D^{\mu} U^{\dagger}+M_{\pi}^{2}\left(U+U^{\dagger}\right)\right], \\
& \mathscr{L}_{\pi N}^{(1)}=N^{\dagger}\left(i v \cdot D+g_{A} S \cdot u\right) N,
\end{aligned}
$$

where the superscript $i$ in $\mathscr{L}^{(i)}$ denotes the number of derivatives and/or pion mass insertions. Here, $F_{\pi}\left(g_{A}\right)$ is the pion decay constant (the nucleon axial-vector coupling), $N$ represents a nucleon field in the heavy-baryon formulation and $S_{\mu}=\frac{1}{2} \gamma_{5} \sigma_{\mu \nu} v^{v}$ is the Pauli-Lubanski spin vector which reduces to $S^{\mu}=\left(0, \frac{1}{2} \vec{\sigma}\right)$ for $v_{\mu}=(1,0,0,0)$. At the order we are working and for the contributions to the current operator considered in the present work, all LECs entering Eq. (2.8) should be taken at their physical values. Further, the $\mathrm{SU}(2)$ matrix $U=u^{2}$ collects the pion fields and various covariant derivatives are defined according to

$$
D_{\mu} U=\partial_{\mu} U-i r_{\mu} U+i U l_{\mu}
$$




$$
\begin{aligned}
u_{\mu} & =i\left[u^{\dagger}\left(\partial_{\mu}-i r_{\mu}\right) u-u\left(\partial_{\mu}-i l_{\mu}\right) u^{\dagger}\right], \\
D_{\mu} N & =\left[\partial_{\mu}+\Gamma_{\mu}-i v_{\mu}^{(s)}\right] N \quad \text { with } \quad \Gamma_{\mu}=\frac{1}{2}\left[u^{\dagger}\left(\partial_{\mu}-i r_{\mu}\right) u+u\left(\partial_{\mu}-i l_{\mu}\right) u^{\dagger}\right] .
\end{aligned}
$$

To describe the coupling to an external electromagnetic field, the left- and right-handed currents $r_{\mu}$ and $l_{\mu}$ and the isoscalar current $v_{\mu}^{(s)}$ have to be chosen as

$$
r_{\mu}=l_{\mu}=\frac{e}{2} \mathscr{A}_{\mu} \tau_{3}, \quad v_{\mu}^{(s)}=\frac{e}{2} \mathscr{A}_{\mu},
$$

where $e$ denotes the elementary charge. Expanding the various terms in the effective Lagrangian in powers of the pion field and using the canonical formalism along the lines of Ref. [17], we end up with the following interaction terms in the Hamilton density

$$
\begin{aligned}
& \mathscr{H}_{21}^{(1)}=\frac{g_{A}}{2 F_{\pi}} N^{\dagger}(\vec{\sigma} \vec{\tau} \cdot \vec{\nabla} \vec{\pi}) N, \\
& \mathscr{H}_{22}^{(2)}=\frac{1}{4 F_{\pi}^{2}} N^{\dagger}[\vec{\pi} \times \dot{\vec{\pi}}] \cdot \vec{\tau} N, \\
& \mathscr{H}_{42}^{(4)}=\frac{1}{32 F_{\pi}^{4}}\left(N^{\dagger}[\vec{\tau} \times \vec{\pi}] N\right) \cdot\left(N^{\dagger}[\vec{\tau} \times \vec{\pi}] N\right),
\end{aligned}
$$

and the electromagnetic current density is of the form

$$
\begin{aligned}
{J_{20}}^{\left({ }^{(-1)}\right.} & =\frac{e}{2} N^{\dagger}\left(\mathbb{1}+\tau_{3}\right) N, \\
{J_{02}}^{(}{ }^{(-1)} & =e[\vec{\pi} \times \dot{\vec{\pi}}]_{3}, \\
\vec{J}_{02}{ }^{(-1)} & =-e[\vec{\pi} \times \vec{\nabla} \vec{\pi}]_{3}, \\
\vec{J}_{21}{ }^{(0)} & =e \frac{g_{A}}{2 F_{\pi}} N^{\dagger} \vec{\sigma}[\vec{\tau} \times \vec{\pi}]_{3} N .
\end{aligned}
$$

In the above expressions we adopt the notation of Ref. [11]. In particular, the subscripts $a$ and $b$ in $\mathscr{H}_{a b}^{(\kappa)}$ and $J_{a b}^{\mu{ }^{(\kappa)}}$ refer to the number of the nucleon and pion fields, respectively, while the superscript $\kappa$ gives the dimension of the operator as defined in Eq. (2.2). Further, the symbol $\cdot \cdot$ in Eq. (2.11) denotes a scalar product in the spin and isospin spaces.

\section{Results in configuration space}

For the sake of convenience, we distinguish between seven classes of contributions according to the power of the $\operatorname{LEC} g_{A}$ (i.e. proportional to $g_{A}^{0}, g_{A}^{2}$ and $g_{A}^{4}$ ) and the type of the hadronic current $J_{20}^{\mu}, J_{21}^{\mu}$ or $J_{02}^{\mu}$ as shown in Fig. 1. Notice that there are no contributions proportional to $g_{A}^{0}$ and involving $J_{20}^{\mu}$ and $J_{21}^{\mu}$. We also emphasize that the second diagram in the class 3 does not generate any contribution. It results from the term in the Hamilton density which is absent in the Lagrangian and arises through the application of the canonical formalism. Finally, it should be understood that the meaning of diagrams in the method of unitary transformation is different from the one arising in the context of covariant and/or time-ordered perturbation theory.

Below, we give explicit results for the current and charge densities, $J^{\mu}=(\rho, \vec{J})$, resulting from the individual classes using the notation

$$
\left\langle\vec{p}_{1}{ }^{\prime} \vec{p}_{2}{ }^{\prime}\left|J^{\mu}\right| \vec{p}_{1} \vec{p}_{2}\right\rangle=\delta\left(\vec{p}_{1}{ }^{\prime}+\vec{p}_{2}{ }^{\prime}-\vec{p}_{1}-\vec{p}_{2}-\vec{k}\right)\left[\sum_{X=\mathrm{c} 1}^{\mathrm{c} 7} J_{\mathrm{X}}^{\mu}+(1 \leftrightarrow 2)\right],
$$




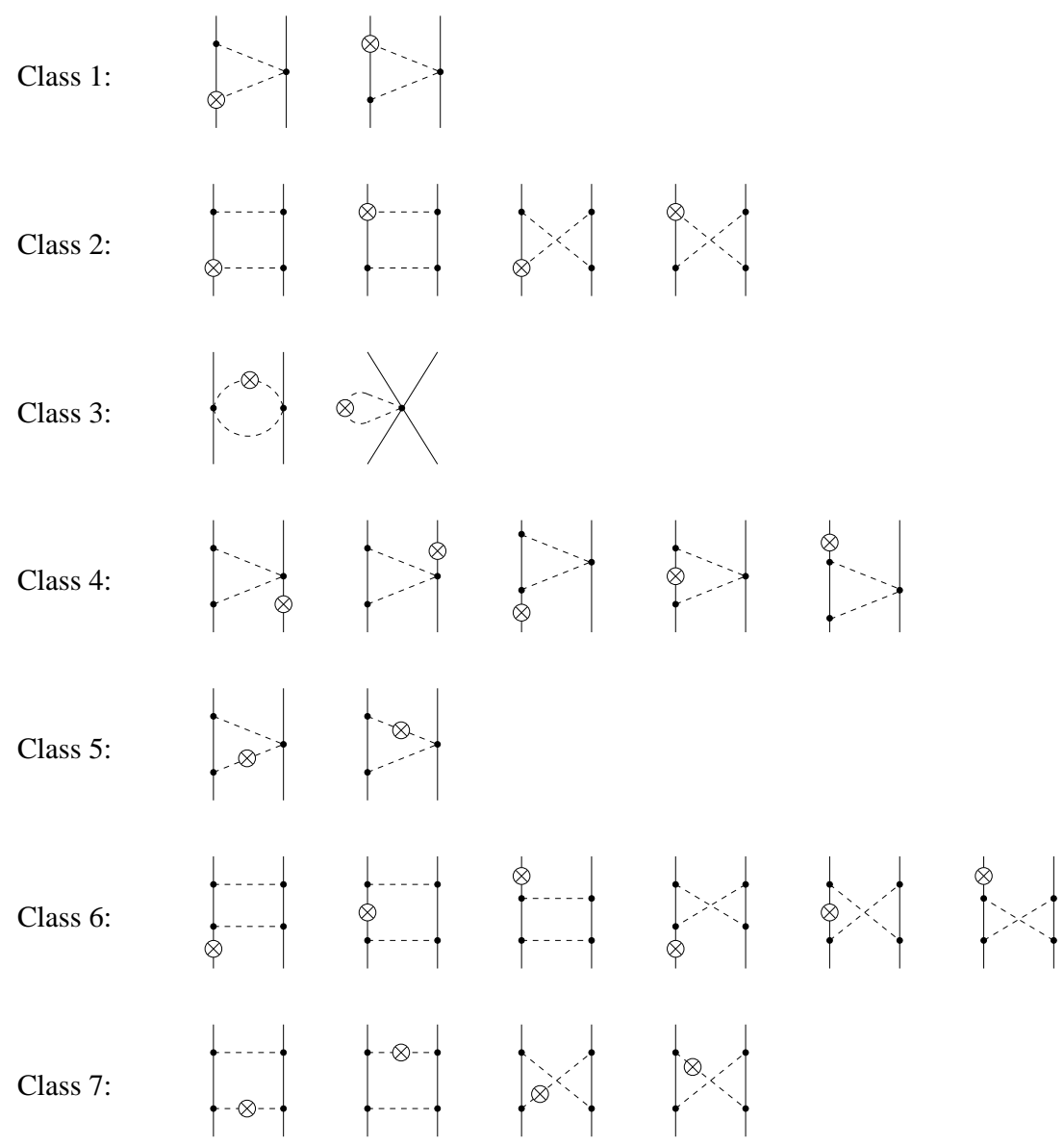

Figure 1: Diagrams showing contributions to the leading two-pion exchange currents. Solid and dashed lines refer to nucleons and pions, respectively. Solid dots are the lowest-order vertices from the effective Lagrangian while the crosses represent insertions of the electromagnetic vertices as explained in the text. Diagrams resulting from interchanging the nucleon lines are not shown.

where $\vec{p}_{i}\left(\vec{p}_{i}{ }^{\prime}\right)$ denotes the incoming (outgoing) momentum of nucleon $i$ and $\vec{k}$ is the photon momentum. In order to avoid confusion with the nucleon labels, in the following we label the Cartesian components of various vectors in isospin space by the superscripts rather than subscripts. Further, $(1 \leftrightarrow 2)$ refers to the contribution resulting from the interchange of the nucleon labels. We find the following compact results for the current density in configuration-space from the individual classes of the diagrams shown in Fig. 1:

$$
\begin{aligned}
\vec{J}_{\mathrm{c} 1}\left(\vec{r}_{10}, \vec{r}_{20}\right)= & e \frac{g_{A}^{2} M_{\pi}^{7}}{128 \pi^{3} F_{\pi}^{4}}\left[\vec{\nabla}_{10}\left[\vec{\tau}_{1} \times \vec{\tau}_{2}\right]^{3}+2\left[\vec{\nabla}_{10} \times \vec{\sigma}_{2}\right] \tau_{1}^{3}\right] \delta\left(\vec{x}_{20}\right) \frac{K_{1}\left(2 x_{10}\right)}{x_{10}^{2}} \\
\vec{J}_{\mathrm{c} 2}\left(\vec{r}_{10}, \vec{r}_{20}\right)= & -e \frac{g_{A}^{4} M_{\pi}^{7}}{256 \pi^{3} F_{\pi}^{4}}\left(3 \nabla_{10}^{2}-8\right)\left[\vec{\nabla}_{10}\left[\vec{\tau}_{1} \times \vec{\tau}_{2}\right]^{3}+2\left[\vec{\nabla}_{10} \times \vec{\sigma}_{2}\right] \tau_{1}^{3}\right] \delta\left(\vec{x}_{20}\right) \frac{K_{0}\left(2 x_{10}\right)}{x_{10}} \\
& +e \frac{g_{A}^{4} M_{\pi}^{7}}{32 \pi^{3} F_{\pi}^{4}}\left[\vec{\nabla}_{10} \times \vec{\sigma}_{1}\right] \tau_{2}^{3} \delta\left(\vec{x}_{20}\right) \frac{K_{1}\left(2 x_{10}\right)}{x_{10}^{2}}
\end{aligned}
$$




$$
\begin{aligned}
\vec{J}_{\mathrm{c} 3}\left(\vec{r}_{10}, \vec{r}_{20}\right)= & -e \frac{M_{\pi}^{7}}{512 \pi^{4} F_{\pi}^{4}}\left[\vec{\tau}_{1} \times \vec{\tau}_{2}\right]^{3}\left(\vec{\nabla}_{10}-\vec{\nabla}_{20}\right) \frac{K_{2}\left(x_{10}+x_{20}+x_{12}\right)}{\left(x_{10} x_{20} x_{12}\right)\left(x_{10}+x_{20}+x_{12}\right)} \\
\vec{J}_{\mathrm{c} 5}\left(\vec{r}_{10}, \vec{r}_{20}\right)= & -e \frac{g_{A}^{2} M_{\pi}^{7}}{256 \pi^{4} F_{\pi}^{4}}\left(\vec{\nabla}_{10}-\vec{\nabla}_{20}\right)\left[\left[\vec{\tau}_{1} \times \vec{\tau}_{2}\right]^{3} \vec{\nabla}_{12} \cdot \vec{\nabla}_{20}-2 \tau_{1}^{3} \vec{\sigma}_{2} \cdot\left[\vec{\nabla}_{12} \times \vec{\nabla}_{20}\right]\right] \\
& \times \frac{K_{1}\left(x_{10}+x_{20}+x_{12}\right)}{\left(x_{10} x_{20} x_{12}\right)}, \\
\vec{J}_{\mathrm{c} 7}\left(\vec{r}_{10}, \vec{r}_{20}\right)= & e \frac{g_{A}^{4} M_{\pi}^{7}}{512 \pi^{4} F_{\pi}^{4}}\left(\vec{\nabla}_{10}-\vec{\nabla}_{20}\right)\left[\left[\vec{\tau}_{1} \times \vec{\tau}_{2}\right]^{3} \vec{\nabla}_{12} \cdot \vec{\nabla}_{10} \vec{\nabla}_{12} \cdot \vec{\nabla}_{20}+4 \tau_{2}^{3} \vec{\sigma}_{1} \cdot\left[\vec{\nabla}_{12} \times \vec{\nabla}_{10}\right] \vec{\nabla}_{12} \cdot \vec{\nabla}_{20}\right] \\
& \times \frac{x_{10}+x_{20}+x_{12}}{x_{10} x_{20} x_{12}} K_{0}\left(x_{10}+x_{20}+x_{12}\right)
\end{aligned}
$$

and the charge density

$$
\begin{aligned}
\rho_{\mathrm{c} 1}\left(\vec{r}_{10}, \vec{r}_{20}\right)= & \rho_{\mathrm{c} 2}\left(\vec{r}_{10}, \vec{r}_{20}\right)=\rho_{\mathrm{c} 3}\left(\vec{r}_{10}, \vec{r}_{20}\right)=0, \\
\rho_{\mathrm{c} 4}\left(\vec{r}_{10}, \vec{r}_{20}\right)= & e \frac{g_{A}^{2} M_{\pi}^{7}}{256 \pi^{2} F_{\pi}^{4}} \tau_{1}^{3} \delta\left(\vec{x}_{20}\right)\left(\nabla_{10}^{2}-2\right) \frac{e^{-2 x_{10}}}{x_{10}^{2}}, \\
\rho_{\mathrm{c} 5}\left(\vec{r}_{10}, \vec{r}_{20}\right)= & -e \frac{g_{A}^{2} M_{\pi}^{7}}{256 \pi^{2} F_{\pi}^{4}} \tau_{2}^{3} \delta\left(\vec{x}_{20}\right)\left(\nabla_{10}^{2}-2\right) \frac{e^{-2 x_{10}}}{x_{10}^{2}}, \\
\rho_{\mathrm{c} 6}\left(\vec{r}_{10}, \vec{r}_{20}\right)= & -e \frac{g_{A}^{4} M_{\pi}^{7}}{256 \pi^{2} F_{\pi}^{4}} \delta\left(\vec{x}_{20}\right)\left[\tau_{1}^{3}\left(2 \nabla_{10}^{2}-4\right)+\tau_{2}^{3} \vec{\sigma}_{1} \cdot \vec{\nabla}_{10} \vec{\sigma}_{2} \cdot \vec{\nabla}_{10}-\tau_{2}^{3} \vec{\sigma}_{1} \cdot \vec{\sigma}_{2}\right] \frac{e^{-2 x_{10}}}{x_{10}^{2}} \\
& -e \frac{g_{A}^{4} M_{\pi}^{7}}{128 \pi^{2} F_{\pi}^{4}} \delta\left(\vec{x}_{20}\right) \tau_{1}^{3}\left(3 \nabla_{10}^{2}-11\right) \frac{e^{-2 x_{10}}}{x_{10}}, \\
\rho_{\mathrm{c} 7}\left(\vec{r}_{10}, \vec{r}_{20}\right)= & -e \frac{g_{A}^{4} M_{\pi}^{7}}{512 \pi^{3} F_{\pi}^{4}}\left[\left(\tau_{1}^{3}+\tau_{2}^{3}\right)\left(\vec{\nabla}_{12} \cdot \vec{\nabla}_{10} \vec{\nabla}_{12} \cdot \vec{\nabla}_{20}+\vec{\nabla}_{12} \cdot\left[\vec{\nabla}_{10} \times \vec{\sigma}_{1}\right] \vec{\nabla}_{12} \cdot\left[\vec{\nabla}_{20} \times \vec{\sigma}_{2}\right]\right)\right. \\
& \left.+\left[\vec{\tau}_{1} \times \vec{\tau}_{2}\right]^{3} \vec{\nabla}_{12} \cdot \vec{\nabla}_{10} \vec{\nabla}_{12} \cdot\left[\vec{\nabla}_{20} \times \vec{\sigma}_{2}\right]\right] \frac{e^{-x_{10}}}{x_{10}} \frac{e^{-x_{20}}}{x_{20}} \frac{e^{-x_{12}}}{x_{12}} .
\end{aligned}
$$

In the above expressions, $K_{n}(x)$ denote the modified Bessel functions of the second kind and we have introduced dimensionless variables $\vec{x}_{10}=M_{\pi} \vec{r}_{10}, \vec{x}_{20}=M_{\pi} \vec{r}_{20}$ and $\vec{x}_{12}=M_{\pi} \vec{r}_{12}=M_{\pi}\left(\vec{r}_{1}-\vec{r}_{2}\right)$. Further, $x_{i j} \equiv\left|\vec{x}_{i j}\right|$ and all derivatives with respect to $\vec{x}_{10}, \vec{x}_{20}$ and $\vec{x}_{12}$ are to be evaluated as if these variables were independent of each other. We also emphasize that the above expressions are valid for $x_{10}+x_{20}>0$. Finally, it should be understood that the behavior of the current and charge densities at short distances will be affected if one uses a regularization with a finite value of the cutoff.

\section{Summary and Outlook}

The application of the method of unitary transformation to derive the leading two-pion exchange two-nucleon charge and current densities based on chiral effective field theory was presented. The resulting nuclear current is given configuration space, where we were able to evaluate all loop integrals analytically leading to very compact expressions in terms of the modified Bessel functions of the second kind. We have also explicitly verified that the derived exchange currents fulfill the continuity equation, see [9] for details. 
In addition to the two-pion exchange contributions, there are also one-pion exchange and shortrange terms at order $\mathscr{O}(e Q)$, see Refs. [7, [] for a recent work based on time-ordered perturbation theory. As will be demonstrated in a subsequent publication [16], renormalization of the one-pion exchange contributions at the one-loop level strongly restricts the ambiguity in the definition of the current and provides a highly non-trivial consistency check of the calculation. In particular, one needs to ensure that all appearing ultraviolet divergences are absorbed into redefinition of the LECs $d_{i}$ and $l_{i}$ from $\mathscr{L}_{\pi N}^{(3)}$ and $\mathscr{L}_{\pi}^{(4)}$, respectively, with already known $\beta$-functions, see e.g. [12, 13, 14, 15]. This work is in progress [16].

Finally, in the future, one also needs to test the convergence of the chiral expansion for the one- and two-pion exchange currents by calculating the corrections at order $\mathscr{O}\left(e Q^{2}\right)$. Given the large numerical values of the LECs $c_{3,4}$ from $\mathscr{L}_{\pi N}^{(2)}$, one might expect sizeable corrections which, indeed, is well known to be the case for the two-pion exchange potential [18]. In this context, it might be advantageous to include the $\Delta(1232)$ isobar as an explicit degree of freedom in effective field theory utilizing the small scale expansion [19].

\section{Acknowledgments}

S.K. would like to thank his collaborators on this subject E. Epelbaum, H. Krebs and U.G. Meißner and the organizers and conveners of this enjoyable conference.

We would also like to thank Walter Glöckle, Jacek Golak, Dagmara Rozpedzik and Henryk Witała for many stimulating discussions on this topic. S.K. would also like to thank Jacek Golak and Henryk Witała for their hospitality during his stay in Krakow where a part of this work was done. This work was supported by funds provided by the Helmholtz Association to the young investigator group "Few-Nucleon Systems in Chiral Effective Field Theory" (grant VH-NG-222) and to the virtual institute "Spin and strong QCD" (VH-VI-231), by the DFG (SFB/TR 16 "Subnuclear Structure of Matter") and by the EU HadronPhysics2 project "Study of strongly interacting matter".

\section{References}

[1] E. Epelbaum, Few-nucleon forces and systems in chiral effective field theory, Prog. Part. Nucl. Phys. 57, 654 (2006), [arXiv: nucl-th/0509032].

[2] J. Golak, R. Skibinski, H. Witala, W. Glöckle, A. Nogga and H. Kamada, Electron and Photon Scattering on Three-Nucleon Bound States, Phys. Rept. 415, 89 (2005), [arXiv:nucl-th/0505072].

[3] E. Epelbaum, W. Glöckle and U.-G. Meißner, The two-nucleon system at next-to-next-to-next-to-leading order, Nucl. Phys. A 747, 362 (2005), [arXiv:nucl-th/0405048].

[4] D. R. Entem and R. Machleidt, Accurate Charge-Dependent Nucleon-Nucleon Potential at Fourth Order of Chiral Perturbation Theory, Phys. Rev. C 68, 041001 (2003), [arXiv:nucl-th/0304018].

[5] E. Epelbaum, A. Nogga, W. Glöckle, H. Kamada, U.-G. Meißner and H. Witala, Three-nucleon forces from chiral effective field theory, Phys. Rev. C 66, 064001 (2002), [arXiv: nucl-th/0208023]. 
[6] E. Epelbaum, H. W. Hammer and U.-G. Meißner, Modern Theory of Nuclear Forces, Rev. Mod. Phys. 81, 1773 (2009), [arXiv:0811.1338 [nucl-th]].

[7] S. Pastore, R. Schiavilla and J. L. Goity, Electromagnetic two-body currents of one-and two-pion range, Phys. Rev. C 78, 064002 (2008), [arXiv:0810.1941 [nucl-th]].

[8] S. Pastore, L. Girlanda, R. Schiavilla, M. Viviani and R. B. Wiringa, Electromagnetic Currents and Magnetic Moments in XEFT, Phys. Rev. C 80, 034004 (2009), [arXiv: 0906.1800 [nucl-th]].

[9] S. Kölling, E.Epelbaum, H. Krebs and U.-G. Meißner, Two-pion exchange electromagnetic current in chiral effective field theory using the method of unitary transformation, Phys. Rev. C 80, 045502 (2009), [arXiv:0907.3437 [nucl-th]].

[10] S. Okubo, Diagonalization of Hamiltonian and Tamm-Dancoff Equation, Prog. Theor. Phys. 12, 603 (1954).

[11] E. Epelbaum, Four-nucleon force using the method of unitary transformation, Eur. Phys. J. A 34, 197 (2007), [arXiv:0710.4250 [nucl-th]].

[12] J. Gasser and H. Leutwyler, Low-Energy Expansion Of Meson Form-Factors, Nucl. Phys. B 250, 517 (1985).

[13] G. Ecker and M. Mojžiš, Low-energy expansion of the pion - nucleon Lagrangian, Phys. Lett. B 365, 312 (1996), [arXiv: hep-ph/9508204].

[14] N. Fettes, U.-G. Meißner and S. Steininger, Pion nucleon scattering in chiral perturbation theory. I: Isospin-symmetric case, Nucl. Phys. A 640, 199 (1998), [arXiv: hep-ph/9803266].

[15] J. Gasser, M. A. Ivanov, E. Lipartia, M. Mojžiš and A. Rusetsky, Ground-state energy of pionic hydrogen to one loop, Eur. Phys. J. C 26, 13 (2002), [arXiv: hep-ph/0206068].

[16] S. Kölling, E. Epelbaum, H. Krebs, U.-G. Meißner, in preparation.

[17] I. S. Gerstein, R. Jackiw, S. Weinberg and B. W. Lee, Chiral loops, Phys. Rev. D 3, 2486 (1971).

[18] N. Kaiser, R. Brockmann and W. Weise, Peripheral nucleon nucleon phase shifts and chiral symmetry, Nucl. Phys. A 625, 758 (1997) [arXiv: nucl-th/9706045].

[19] T. R. Hemmert, B. R. Holstein, and J. Kambor, Chiral Lagrangians and delta(1232) interactions: Formalism, J. Phys. G 24, 1831 (1998), [arXiv: hep-ph/9712496]. 